\title{
High Sensitive CRP Level Is Associated With Intermediate and High Syntax Score in Patients With Acute Coronary Syndrome
}

\author{
Muhammed Karadeniz, ${ }^{1}$ MD, Mustafa Duran, ${ }^{1}$ MD, Ahmet AKyel, ${ }^{1}$ MD, \\ Mikail YARlıŏ́Lueș, ${ }^{1}$ MD, Adil Hakan ÖCeK, ${ }^{1}$ MD, İbrahim Etem ÇElıK, ${ }^{1}$ MD, Alparslan KıLıç, ${ }^{1}$ MD, \\ Ahmet Arif Yalcin, ${ }^{2}$ MD, Gökhan ErgüN, ${ }^{1}$ MD, and Sani Namık Murat, ${ }^{1}$ MD
}

\section{SUMMARY}

High sensitive C-reactive protein (hs-CRP) levels are associated with short- and long-term mortality in patients with acute coronary syndrome (ACS). We investigated whether baseline hs-CRP levels are associated with burden of coronary atherosclerosis assessed by SYNTAX score (SXScore).

We enrolled 321 patients with ACS who underwent coronary angiography. The patients were divided into tertiles according to the SXScore: low SXScore $(\leq 22)$, and intermediate-high SXScore $(\geq 23)$.

Subjects in the intermediate-high SXScore tertile had higher serum hs-CRP levels compare to low SXScore tertile patients $(7.7 \pm 3.4 \mathrm{mg} / \mathrm{L}$ versus $4.9 \pm 2.5 \mathrm{mg} / \mathrm{L}, P<0.001)$. The mean age of patients and prevalance of diabetes in the intermediate-high SXScore tertile were significantly higher than in the low SXScore tertile (63 \pm 13 versus $58 \pm 12$ years $P=0.001$ for age, $P=0.007$ for diabetes). Multivariate logistic regression analysis showed that the strongest predictors of high SXScore were increased serum hs-CRP levels (OR: 1.14) together with multivessel disease (OR: 0.23), left ventricular ejection fraction (LVEF) (OR: 0.90), and troponin levels (OR: 1.12).

Serum hs-CRP levels on admission in patients with ACS could predict the severity and complexity of coronary atherosclerosis together with multivessel disease, LVEF, and troponin levels. Thus, increased serum levels of hs-CRP were one of the strong predictors of high SXScore in ACS patients. (Int Heart J 2015; 56: 377-380)

Key words: Inflammation, Severity of atherosclerosis, Coronary artery disease

$\mathrm{T}$ The SYNTAX score (SXScore) is a useful angiographic scoring system and it can be used to cumulatively quantify the extent, severity, and complexity of coronary artery disease $(\mathrm{CAD}) .^{1-3)}$ The SXScore is an independent predictor of long-term mortality and morbidity in several patient types including acute coronary syndrome (ACS) ${ }^{4.8)}$

Inflammation plays an important role in the atherosclerotic process at the beginning of endothelial dysfunction through all stages of plaque formation until its detrimental clinical ischemic complications. ${ }^{9,10}$ Elevated levels of high sensitivity C-reactive protein (hs-CRP), a well known acutephase protein and an extremely sensitive marker of systemic inflammation, is also an important risk factor for atherosclerosis and CAD ${ }^{6,7)}$ Several studies concluded that hs-CRP levels were a useful marker to predict further cardiovascular events in patients with CAD. ${ }^{11,12)}$

In this study, we aimed to investigate whether hs-CRP was associated with the SXScore in patients with ACS.

\begin{abstract}
MethodS
Study population: We enrolled 321 patients with ACS who underwent coronary angiography between January 2013 and November 2013. The clinical spectrum of ACS consists of ST elevation myocardial infarction (STEMI, $n=224,69.8 \%$ ) and nonST elevation acute coronary syndromes [NST-ACS, including non ST elevation myocardial infarction (NSTEMI) or unstable angina pectoris (USAP), $n=97,30.2 \%$ ], which are classified from the acute phase electrocardiography (ECG) changes and the development of myocardial necrosis. Patients with ACS, an SXScore of zero, history of prior coronary artery bypass grafting surgery $(\mathrm{CABG})$, cardiopulmonary resuscitation before admission, presence of advanced valve disease and advanced renal/hepatic disease, known malignancy, a history of congestive heart failure, and/or the presence of overt pump failure ( $\geq$ Killip class III) on admission were excluded from the study.

Diagnostic criteria for STEMI and NSTE-ACS: The leading symptom of ACS is typically chest pain. Patients with ST segment elevation in $\geq 2$ consecutive leads (with the cut-off point
\end{abstract}

From the ${ }^{1}$ Department of Cardiology, Ankara Education and Research Hospital, Ankara, ${ }^{2}$ Department of Cardiology, Mehmet Akif Ersoy Thoracic and Cardiovascular Surgery, Training and Research Hospital, Istanbul, Turkey.

Address for correspondence: Muhammed Karadeniz, MD, Department of Cardiology, Ankara Education and Research Hospital, Sukriye Mh. Ulucanlar Cad. No:89 Altindağ, Ankara, Turkey. E-mail: mduran2@gmail.com

Received for publication September 17, 2014. Revised and accepted February 2, 2015.

Released in advance online on J-STAGE June 26, 2015.

All rights reserved by the International Heart Journal Association. 
being $>0.2 \mathrm{mV}$ in leads $\mathrm{V} 1, \mathrm{~V} 2$, or $\mathrm{V} 3$, and $>0.1 \mathrm{mV}$ in the other leads) were defined as having STEMI. The working diagnosis of NSTE-ACS is a rule-out diagnosis based on the ECG, ie, lack of persistent ST elevation. Biomarkers (troponins) further distinguish NSTEMI and USAP. Imaging modalities are used to rule out or rule in differential diagnoses. The clinical presentation of NSTE-ACS encompasses a wide variety of symptoms. Traditionally, several clinical presentations have been distinguished: Prolonged ( $>20$ minutes) anginal pain at rest; New onset (de novo) angina (Class II or III of the Classification of the Canadian Cardiovascular Society); Recent destabilization of previously stable angina with at least Canadian Cardiovascular Society Class III angina characteristics (crescendo angina); or Post-MI angina. The characteristic ECG abnormalities of NSTE-ACS are ST-segment depression or transient elevation and/or T-wave change. At presentation, the working diagnosis of NSTE-ACS, based on the measurement of troponins, will be further qualified as NSTEMI or USAP. Patients with normal troponin levels were accepted as USAP. Patients with high troponin levels were accepted as NSTEMI.

The study protocol was approved by the local ethics committee and all patients gave their written informed consent.

hs-CRP analysis: Serum hs-CRP levels were measured from blood samples taken at admission to the emergency service or catheter laboratory. Creatinine clearance was also calculated from plasma creatinine levels assessed from blood samples taken at about one hour after first contact. ${ }^{13)}$

Coronary angiography and SXScore analysis: Coronary angiography was performed by the Judkins technique (Siemens Axiom Artis zee 2011; Siemens Healthcare, Erlangen, Germany). The severity and complexity of coronary atherosclerosis was assessed in several ways. The first was a simple classification in number of diseased vessel scoring system. The number of diseased vessels with $\geq 50 \%$ luminal stenosis in major coronary arteries was scored as 1 to 3 diseased vessels. In addition, angiograms were scored according to the SXScore system. ${ }^{2,3)}$ All lesions causing $\geq 50 \%$ stenosis in a coronary artery with a diameter $\geq 1.5 \mathrm{~mm}$ were included in the SXScore calculation. For the calculation, the software on the website (http://www. syntaxscore.com) was used. SXscore was evaluated separately by 2 interventional cardiologists blinded to the study protocol and patient characteristics. In the presence of a contradiction between the two results, the opinion of a senior interventional cardiologist was applied and a common consensus was obtained. The patients were divided into tertiles according to the SXScore: low SXScore $(\leq 22)$, and intermediate-high SXScore $(\geq 23)$.

Transthoracic echocardiography (TTE) was performed for all patients within 48 hours after hospitalisation (Vivid 3; GE Medical System, Horten, Norway). Left ventricular ejection fraction (LVEF) was measurement using the Simpson method.

Statistical analysis: All analyses were performed using SPSS for Windows (version 18.0, SPSS, Chicago, Illinois, USA). Quantitative variables are expressed as the mean value \pm SD for parametric variables, and median and minimum-maximum levels for non-parametric variables. Continuous variables were analyzed for normal distribution using the Kolmogorov-Smirnov test and analyzed for homogeneity using the Levene tests. Comparisons of parametric values among groups were performed by the Student $t$ test. Categorical variables were compared with the chi-square test. A two-tailed $P<0.05$ was con- sidered significant. Univariate logistic regression was used to identify independent predictors of a high SXScore [variables: age, diabetes mellitus, multivessel disease, LVEF, Killip class, troponin, uric acid, hs-CRP, LDL cholesterol levels]. After performing univariate analysis, significantly obtained variables (multivessel disease, LVEF, troponin and hs-CRP levels) were used in multivariate logistic regression analysis. Receiver operating characteristic (ROC) curve analysis was performed in order to determine the best cutoff value of hs-CRP and the sensitivity and specificity at that point were obtained for predicting a high SXSscore tertile.

\section{Results}

From the initial 359 consecutive patients admitted, 38 were excluded due to various exclusion criteria. A total of 321 patients matched the inclusion and exclusion criteria. The baseline characteristics of the study population classified according to SXScore tertiles are presented in Supplemental Table I. The mean age of patients in the intermediate-high SXScore tertile was significantly higher than the low SXScore tertile ( $63 \pm 13,58 \pm 12$ years respectively, $P=0.001$ for both). Diabetes prevalence was higher in the intermediate-high SXScore tertile than the low SXScore tertile $(P=0.007)$. Additionally, LVEF was significantly lower in the patients with an intermediate-high SXScore compared to low SXScore tertiles $(P$ $<0.001)$. Patients with an intermediate-high SXScore more commonly presented with STEMI, while patients with low tertile more commonly presented with NSTE-ACS.

Laboratory measurements of the patients stratified into SXScore tertiles are shown in Supplemental Table II. Subjects in the intermediate-high SXScore tertile had higher serum hsCRP compared to the low SXScore tertile (mean hs-CRP levels were $7.7 \pm 3.4 \mathrm{mg} / \mathrm{L}$ versus $4.9 \pm 2.5 \mathrm{mg} / \mathrm{L}, P<0.001)$. Furthermore, patients in the intermediate-high SXScore tertile had significantly higher serum glucose, creatinine, peak creatine kinase muscle and brain (CK-MB), peak troponin, and uric acid levels, while they had lower values of estimated glomerular filtration rate (eGFR), hemoglobin, total cholesterol, and LDL cholesterol in comparison to subjects in the lower tertiles (for $P$-values see Supplemental Table II). We found a significant relationship between hs-CRP and SXScore with correlation analysis $(r=0.361, P<0.001)$.

Angiographic and procedural characteristics, and the management of patients according to SXscore tertiles are presented in Supplemental Table III. Patients with an intermediate-high SXScore had a significantly higher rate of multivessel disease and chronic total occlusion compared to low SXScore patients $(P<0.001$ for both). Single, two, and three vessel diseases were detected in $118(55 \%), 66(31 \%)$, and $30(14 \%)$, respectively, in low SXScore patients and 27 (26\%), 34 (33\%), and $42(41 \%)$, respectively, in intermediate-high SXScore patients $(P<0.001)$. The left anterior descending artery was more commonly the culprit vessel in the intermediate-high SXScore tertile and the right coronary artery was more commonly the culprit vessel in the low SXScore tertile. The rate of direct stenting was higher in patients with a low SXScore, while a balloon with stenting was more common in patients with an intermediate-high SXScore.

Univariate logistic regression analysis was used to identi- 
fy independent predictors of intermediate-high SXScore tertile (variables: age, diabetes mellitus, multivessel disease, LVEF, Killip class, troponin, uric acid, hs-CRP, LDL cholesterol levels). After performing univariate analysis, significant variables (multivessel disease, LVEF, troponin and hs-CRP levels) were used in multivariate logistic regression analysis. Multivariate logistic regression analysis showed that the strongest predictors of a high SXScore were increased serum hs-CRP levels (OR: 1.14, 95\%CI: 1.05-1.25, $P=0.002$ ) together with multivessel disease (OR: $0.23,95 \% \mathrm{CI}: 0.12-0.44, P<0.001), \mathrm{LVEF}$ (OR: 0.90, 95\%CI: 0.86-0.93, $P<0.001$ ), and troponin levels (OR: 1.12, 95\%CI: 1.05-1.27, $P=0.001$ ) (Supplemental Table IV). Thus, increased serum levels of hs-CRP were a strong predictor of a high SXScore in ACS patients. We also used the Syntax score as a numerical variable in the multivariate analysis in Supplemental Table V. According to this analysis, age, hsCRP, diabetes, Killip score, and creatinine were independent predictors of a high SXScore.

ROC curve analysis showed that hs-CRP at a cut-off point of $5.77 \mathrm{mg} / \mathrm{L}$ was highly sensitive $(75 \%)$ and specific (69\%) and was a strongly independent predictor of a high SXScore in patients with ACS (AUC $=0.775, P<0.001$ ) (Supplemental Figure).

\section{Discussion}

One of the most important results in this study was that serum hs-CRP levels on admission in patients with ACS could predict the severity and complexity of coronary atherosclerosis together with multivessel disease, LVEF, and troponin levels. In patients with ACS, several clinical and laboratory parameters and risk scores have been proven to be useful for short and long-term risk assessment. ${ }^{14)}$ However, despite these available parameters and scores, there are still insufficiencies in determining the risk assessment of patients with ACS. Numerous biomarkers, especially chronic inflammation markers, have been investigated for determining the risk assessment of ACS patients.

An association between hs-CRP and cardiovascular events in patients with stable and unstable ACS patients was reported in several studies. The authors showed in the ULSAM study that hs-CRP was an independent predictor of major fatal cardiovascular events and total mortality even in the presence of other important biomarkers such as troponin I and N-terminal pro- brain natriuretic peptide (NT-proBNP). ${ }^{15)}$ The Emerging Risk Factors Collaboration study reported in a meta-analysis that hs-CRP was an independent predictor of major vascular outcomes in predicting future events and hs-CRP performed equally well or better than several traditional risk factors. ${ }^{16)}$ In several studies, it was reported that elevated hs-CRP on admission was found to be a significant predictor of longterm and short-term mortality in patients with USAP and NSTACS. ${ }^{17-20)}$ Haverkate, et al reported that an increase in hs-CRP was associated with a 50\% increase in the risk for a coronary event in patients with stable CAD. ${ }^{17)}$ Assadpour Piranfar and Habib, et al reported that hsCRP correlated with the severity of atherosclerosis but they used only the Gensini score for assessing the severity of atherosclerosis. ${ }^{21,22)}$ In our study, we used the SXScore (which was more sensitive than the Gensini score) to assess the severity of atherosclerosis. Another differ- ence of our study from other studies is that as the SXScore is quantitative data, we investigated the relationship between the SXScore and hsCRP value when they were analyzed with multiple regression analysis. According to the multiple regression analysis in Supplemental Table V, because the standardized coefficient of hsCRP (0.297) was larger than the other variables, we could confirm that hsCRP was a strong predictive value for the SXScore. We believe it is important to demonstrate that the hsCRP level is a predictive indicator not only for an intermediate and high SXScore ( $\geqq 22$ ), but also for the SXScore value itself.

Chronic inflammation plays a pivotal role in the initiation and progression of atherosclerosis. ${ }^{23,24)}$ hs-CRP is a classical acute-phase protein, and reflects systemic inflammation and improved risk prediction for patients with CAD, including ACS. ${ }^{14)}$ According to the Physicians' Health Study results, hsCRP level was a better predictor of vascular events than several other inflammatory biomarkers. ${ }^{25)}$ hs-CRP levels were found to be associated with atherosclerotic plaque instability and a sign of increased risk of acute coronary events. ${ }^{26}$

Our study supports the hypothesis that increasing levels of hs-CRP in patients with ACS may play a role in the severity of CAD. Although the physiopathogenesis of the association between elevated hs-CRP and severity of CAD is not completely understood, multiple mechanisms may be involved. First of all, the association between hs-CRP and severity of CAD could be explained by an association between inflammation and atherosclerosis. Suzuki, et al reported that a positive correlation between serum CRP levels and coronary plaque area suggested a relationship between systemic inflammation and the severity of CAD. ${ }^{27)}$ Secondly, hs-CRP has been shown to impair the endothelial progenitor cells, impair fibrinolysis, increase collagen degradation in monocytes, and activate the complement system. ${ }^{28)}$ Thirdly, it has also been shown that hsCRP may be involved in the uptake of LDL-C by macrophages and turning them into foam cells which are the most important stages for the initiation and progress of atherosclerosis. ${ }^{29,30)}$ These mechanisms may contribute to understanding the relationship between hs-CRP and severity of atherosclerosis. Our study showed for the first time a positive association between hs-CRP and severity of CAD by SXScore in patients with ACS.

Previous reports showed that there is a close relationship between the severity of atherosclerosis and advanced age, presence of diabetes, high levels of uric acid, creatinine, and low levels of estimated glomerular filtration rate (eGFR), hemoglobin, and LVEF. ${ }^{31-34)}$ Similar to these results, we found a significant relationship between the severity of atherosclerosis and advanced age, presence of diabetes, low levels of LVEF, eGFR, and hemoglobin and high levels of creatinine, glucose, and uric acid.

Hs-CRP can be considered as an excellent indicator of the total inflammatory burden of the body and is associated with many cardiovascular traditional risk factors. Therefore, a simple and inexpensive measurement method for hs-CRP levels in the setting of cardiovascular risk evaluation may provide a more comprehensive view of the total risk profile of a patient and seems to be associated with adverse outcome independent of traditional risk factors in patients with CAD.

Study limitations: The assessment of coronary angiographic findings was limited to visual interpretation and angiography is a technique that detects only major coronary arterial lesions. 
Conclusion: Serum hs-CRP levels on admission were independently associated with the extent, severity, and complexity of coronary atherosclerosis in patients with ACS together with LVEF, multivessel disease, and troponin. To the best of our knowledge, this is the first study to demonstrate that hs-CRP is highly sensitive and specific, and is a significant independent predictor of a high SXScore in patients with ACS. Thus, hsCRP assessment may be considered in clinical practice for early risk stratification of patients with ACS.

\section{Disclosure}

Statement of competing interests: The authors report no competing interests.

\section{REFERENCES}

1. Sianos G, Morel MA, Kappetein AP, et al. The SYNTAX Score: an angiographic tool grading the complexity of coronary artery disease. EuroIntervention 2005; 1: 219-27.

2. Serruys PW, Onuma Y, Garg S, et al. Assessment of the SYNTAX score in the Syntax study. EuroIntervention 2009; 5: 50-6.

3. SYNTAX Working Group. SYNTAX score calculator. Available at: http://www.syntaxscore.com. Accessed April 8, 2015.

4. Palmerini T, Genereux P, Caixeta A, et al. Prognostic value of the SYNTAX score in patients with acute coronary syndromes undergoing percutaneous coronary intervention: analysis from the ACUITY (Acute Catheterization and Urgent Intervention Triage StrategY) trial. J Am Coll Cardiol 2011; 57: 2389-97.

5. Valgimigli M, Serruys PW, Tsuchida K, et al. Cyphering the complexity of coronary artery disease using the syntax score to predict clinical outcome in patients with three-vessel lumen obstruction undergoing percutaneous coronary intervention. Am J Cardiol 2007; 99: 1072-81.

6. Capodanno D, Di Salvo ME, Cincotta G, Miano M, Tamburino C, Tamburino C. Usefulness of the SYNTAX score for predicting clinical outcome after percutaneous coronary intervention of unprotected left main coronary artery disease. Circ Cardiovasc Interv 2009; $2: 302-8$

7. Huang G, Zhao JL, Du H, Lan XB, Yin YH. Coronary score adds prognostic information for patients with acute coronary syndrome. Circ J 2010; 74: 490-5.

8. van Gaal WJ, Ponnuthurai FA, Selvanayagam J, et al. The Syntax score predicts peri-procedural myocardial necrosis during percutaneous coronary intervention. Int J Cardiol 2009; 135: 60-5.

9. Halkin A, Singh M, Nikolsky E, et al. Prediction of mortality after primary percutaneous coronary intervention for acute myocardial infarction: the CADILLAC risk score. J Am Coll Cardiol 2005; 45: 1397-405.

10. Yan AT, Yan RT, Tan M, et al. In-hospital revascularization and one-year outcome of acute coronary syndrome patients stratified by the GRACE risk score. Am J Cardiol 2005; 96: 913-6.

11. de Lemos JA, McGuire DK, Drazner MH. B-type natriuretic peptide in cardiovascular disease. Lancet 2003; 362: 316-22.

12. Weber M, Dill T, Arnold R, et al. N-terminal B-type natriuretic peptide predicts extent of coronary artery disease and ischemia in patients with stable angina pectoris. Am Heart J 2004; 148: 612-20.

13. Cockcroft DW, Gault MH. Prediction of creatinine clearance from serum creatinine. Nephron 1976; 16: 31-41.

14. Koenig W. High-sensitivity C-reactive protein and atherosclerotic disease: from improved risk prediction to risk-guided therapy. Int $\mathrm{J}$ Cardiol 2013; 168: 5126-34. (Review)

15. Zethelius B, Berglund L, Sundström J, et al. Use of multiple biomarkers to improve the prediction of death fromcardiovascular causes. N Engl JMed 2008; 358: 2107-16.
16. Emerging Risk Factors Collaboration, Kaptoge S, Di Angelantonio E, Lowe G, et al. C-reactive protein concentration and risk of coronary heart disease, stroke, and mortality: an individual participant meta-analysis. Lancet 2010; 375: 132-40.

17. Haverkate F, Thompson SG, Pyke SD, Gallimore JR, Pepys MB. Production of C-reactive protein and risk of coronary events in stable and unstable angina. Lancet 1997; 349: 462-6.

18. Lindahl B, Toss H, Siegbahn A, Venge P, Wallentin L. Markers of myocardial damage and inflammation in relation to long-term mortality in unstable coronary artery disease. FRISC Study Group. Fragmin during Instability in Coronary Artery Disease. N Engl J Med 2000; 343: 1139-47.

19. Mueller C, Buettner HJ, Hodgson JM, et al. Inflammation and long-term mortality after non-ST elevation acute coronary syndrome treated with a very early invasive strategy in 1042 consecutive patients. Circulation 2002; 105: 1412-5.

20. Bogaty P, Boyer L, Simard S, et al. Clinical utility of C-reactive protein measured at admission, hospital discharge, and 1 month later to predict outcome in patients with acute coronary disease. The RISCA (recurrence and inflammation in the acute coronary syndromes) study. J Am Coll Cardiol 2008; 51: 2339-46.

21. Assadpour Piranfar M. The Correlation between High-Sensitivity C-Reactive Protein (hsCRP) Serum Levels and Severity of Coronary Atherosclerosis. Int Cardiovasc Res J 2014; 8: 6-8.

22. Habib SS, A Al Masri A. Relationship of high sensitivity C-reactive protein with presence and severity of coronary artery disease. Pak J Med Sci 2013; 29: 1425-9.

23. Ross R. Atherosclerosis--an inflammatory disease. N Engl J Med 1999; 340: 115-26. (Review)

24. Hansson GK. Inflammation, atherosclerosis, and coronary artery disease. N Engl J Med 2005; 352: 1685-95. (Review)

25. Ridker PM, Brown NJ, Vaughan DE, Harrison DG, Mehta JL. Established and emerging plasma biomarkers in the prediction of first atherothrombotic events. Circulation 2004; 109: IV6-19. (Review)

26. Abhashi SA, Kryeziu FU, Nazreku FD. Increased carotid intimamedia thickness associated with high hs-CRP levels is a predictor of unstable coronary artery disease. Cardiovasc J Afr 2013; 24 : 270-3.

27. Suzuki M, Saito M, Nagai T, Saeki H, Kazatani Y. Systemic versus coronary levels of inflammation in acute coronary syndromes. Angiology 2006; 57: 459-63.

28. Bisoendial RJ, Boekholdt SM, Vergeer M, Stroes ES, Kastelein JJ. C-reactive protein is a mediator of cardiovascular disease. Eur Heart J 2010; 31: 2087-91. (Review)

29. Jialal I, Devaraj S, Venugopal SK. C-reactive protein: risk marker or mediator in atherothrombosis. Hypertension 2004; 44: 6-11. (Review)

30. Devaraj S, Singh U, Jialal I. The evolving role of C-reactive protein in atherothrombosis. Clin Chem 2009; 55: 229-38. (Review)

31. Duran M, Uysal OK, Gunebakmaz O, et al. Glomerular filtration rate is associated with burden of coronary atherosclerosis in patients with acute coronary syndrome. Angiology 2014; 65: 350-6.

32. Duran M, Uysal OK, Yilmaz Y, et al. Serum gamma-glutamyltransferase and the burden of atherosclerosis in patients with acute coronary syndrome. Turk Kardiyol Dern Ars 2013; 41: 275-81.

33. Duran M, Kalay N, Akpek M, et al. High levels of serum uric acid predict severity of coronary artery disease in patients with acute coronary syndrome. Angiology 2012; 63: 448-52.

34. Aksakal E, Tanboga IH, Kurt M, et al. Predictors of coronary lesions complexity in patients with stable coronary artery disease. Angiology 2013; 64: 304-9.

\section{SuPPlemental FiLes}

Supplemental Table I, II, III, IV, V

Supplemental Figure

Please find supplemental files;

https://www.jstage.jst.co.jp/article/ihj/56/4/56_14-299/_article/supplement 